\title{
CARACTERÍSTICAS DOS IDOSOS SUBMETIDOS AOS EXAMES PARA A CARTEIRA DE HABILITAÇÃO VEICULAR
}

\author{
Maria Helena Lenardt ${ }^{1}$, Nathalia Hammerschmidt Kolb Carneiro ${ }^{2}$, Maria Angélica Binotto ${ }^{3}$, Clovis Cechinel ${ }^{4}$, \\ Tânia Maria Lourenço ${ }^{5}$, Letícia Marie Sakai ${ }^{6}$
}

RESUMO: Trata-se de estudo quantitativo descritivo retrospectivo, cujo objetivo foi rastrear as características dos idosos que realizam o exame de aptidão física e mental para a carteira nacional de habilitação veicular. Coletaram-se dados em 1023 formulários de idosos com idade $\geq 60$ anos em uma clínica de medicina do tráfego na cidade de Curitiba-PR. Houve predomínio de homens $(n=713 ; 69,7 \%)$, idade entre 60 e 64,9 anos $(n=418$; $40,9 \%)$, uso de medicamentos ( $n=621 ; 60,7 \%)$, sobrepeso $(n=419 ; 41 \%)$ e acuidade visual diminuída $(n=573 ; 56 \%)$. Quanto à aptidão para dirigir, $216(21,1 \%)$ foram considerados aptos; 803 (78,5\%) aptos com restrição e quatro $(0,4 \%)$ inaptos temporários. A maioria dos idosos motoristas é idoso-jovem, do sexo masculino e que possui restrição para dirigir pelo uso de lentes oculares. Os resultados fornecem subsídios para o avanço da temática em estudos que têm por objetivo aprimorar as avaliações dos idosos e, consequentemente, contribuir para um trânsito mais seguro.

DESCRITORES: Idoso; Exame para Habilitação de Motoristas; Condução de Veículo; Enfermagem Geriátrica.

\section{CHARACTERISTICS OF ELDERLY SUBMITTED TO DRIVER LICENSE TESTS}

\begin{abstract}
A quantitative, descriptive and retrospective study was undertaken to screen for the characteristics of elderly people who took the physical and mental aptitude test to get the Brazilian driver license. Data were collected from 1023 forms of elderly aged $\geq 60$ years at a clinic for traffic medicine in Curitiba-PR. The predominant characteristics were men $(n=713 ; 69.7 \%)$, between 60 and 64.9 years of age $(n=418 ; 40.9 \%)$, medicine use $(n=621 ; 60.7 \%)$, overweight $(n=419 ; 41 \%)$ and reduced visual acuity $(n=573 ; 56 \%)$. As regards the aptitude to drive, $216(21.1 \%)$ were considered apt; 803 (78.5\%) apt with restriction and four (0.4\%) temporarily inapt Most elderly drivers are young elderly, male with restriction to drive due to the use of corrective lenses. The results offer support to advance on the theme in studies intended to improve the elderly's assessments and, consequently, contribute to safer traffic.
\end{abstract}

DESCRIPTORS: Aged; Automobile Driver Examination; Automobile Driving; Geriatric Nursing.

\section{CARACTERÍSTICAS DOS IDOSOS SUBMETIDOS AOS EXAMES PARA A CARTEIRA DE HABILITAÇÃO VEICULAR}

RESUMEN: Se trata de estudio cuantitativa descriptivo retrospectivo con objeto de rastrear las características de los ancianos que realizan el examen de aptitud física y mental para la conducción de vehículos. Fueron recolectados datos en 1023 formularios de ancianos con edad $\geq 60$ años en una clínica de medicina del tránsito en la ciudad de Curitiba-PR. Predominaron hombres ( $\mathrm{n}=713$; $69,7 \%$ ), edad entre 60 y 64,9 años $(n=418 ; 40,9 \%)$, uso de medicamentos ( $n=621 ; 60,7 \%)$, sobrepeso ( $n=419 ; 41 \%)$ y acuidad visual disminuida ( $\mathrm{n}=573 ; 56 \%)$. Respecto a la aptitud para dirigir, $216(21,1 \%)$ fueron considerados aptos; 803 (78,5\%) aptos con restricción y cuatro $(0,4 \%)$ inaptos temporarios. La mayoría de los ancianos motoristas es anciano-joven, del sexo masculino y con restricción para dirigir debido al uso de lentes oculares. Los resultados fornecen subsidios para el avance del tema en estudios con objeto de perfeccionar las evaluaciones de los ancianos y, consecuentemente, contribuir hacia un tránsito más seguro.

DESCRIPTORES: Anciano; Examen de Aptitud para la Conducción de Vehículos; Conducción de Automóvil; Enfermería Geriátrica.

${ }^{1}$ Enfermeira. Doutora em Filosofia da Enfermagem. Docente do Programa de Pós-Graduação em Enfermagem da Universidade Federal do Paraná. Curitiba, PR, Brasil.

${ }^{2}$ Enfermeira. Doutoranda em Enfermagem. Universidade Federal do Paraná. Curitiba, PR, Brasil.

${ }^{3}$ Educadora Física. Doutoranda em Enfermagem. Universidade Federal do Paraná. Curitiba, PR, Brasil.

${ }^{4}$ Médico. Mestre em Enfermagem. Médico Geriatra do Hospital Zilda Arns. Curitiba, PR, Brasil.

${ }^{5}$ Enfermeira. Doutoranda em Enfermagem. Universidade Federal do Paraná. Curitiba, PR, Brasil.

${ }^{6}$ Discente de Enfermagem. Universidade Federal do Paraná. Curitiba, PR, Brasil.

Autor Correspondente:

Nathalia Hammerschmidt Kolb Carneiro

Universidade Federal do Paraná

R. Lothario Meissner, 632 - 80210-170 - Curitiba, PR, Brasil

E-mail: nathalia.kolb@gmail.com
Recebido: 23/08/2016

Finalizado: 07/02/2017 


\section{INTRODUÇÃO}

Quando se considera o envelhecimento e longevidade, muitas são as preocupações acerca dessas mudanças e do seu impacto sobre a qualidade de vida das pessoas, o que, apesar de ser uma conquista, gera vários desafios para o sujeito que envelhece e para a sociedade em geral.

Para que o envelhecimento seja uma experiência positiva, uma vida mais longa deve ser acompanhada de oportunidades contínuas de saúde, participação e segurança ${ }^{(1)}$. Nessas circunstâncias, podese destacar a necessidade de boas condições de mobilidade, o que remete ao tema transporte e à condução de veículos automotores pelos idosos.

De acordo com dados da Pesquisa Nacional de Viagem da Inglaterra, a percentagem de pessoas com 70 anos ou mais com carteira de habilitação aumentou de 38\% em 1995-1997 para 58\% em 2012. Dado este mais evidente entre as mulheres com 70 anos ou mais, em que o número de habilitações dobrou de 21\% em 1995-1997 para 42\% em 2012. No mesmo período, nos idosos com 60 a 69 anos, observou-se um aumentou de $63 \%$ para $79 \%$ nos homens e de $45 \%$ a $70 \%$ nas mulheres no mesmo período ${ }^{(2)}$.

Levantamento realizado pelo Departamento de Trânsito no estado do Paraná revelou que 219.439 motoristas com mais de 65 anos estão ativos e dirigindo no estado. Eles representam 4,7\% dos 4,5 milhões de condutores paranaenses registrados e a tendência é que este percentual aumente nos próximos anos ${ }^{(3)}$.

O conceito de segurança no trânsito e suas implicações para a manutenção da mobilidade das pessoas idosas são temas emergentes, no entanto requerem pressa na efetividade, em razão do aumento significativo da frota de automóveis e motocicletas e da violência no trânsito. A manutenção da direção veicular ao idoso equivale à independência e liberdade, além do acesso social e às atividades de lazer, que constituem importantes fatores que previnem o isolamento de idosos. Ainda, diminui a chance de institucionalização e de depressão nesses indivíduos ${ }^{(4)}$. Todavia, a direção veicular é uma função complexa que requer a integração dos órgãos sensoriais, da função cognitiva e da atividade psicomotora. Os condutores devem, de forma contínua, perceber as mudanças no seu meio, tomar as decisões baseadas nessas percepções e executar as respostas apropriadas ${ }^{(5)}$.

Alguns fatores podem contribuir para que os idosos se tornem incapazes de dirigir, tais como a perda da visão, audição, força muscular, flexibilidade, excesso de multas e dificuldade para realizar as provas de avaliação(6).

O uso de medicamentos, os índices de doenças e fragilidade física contribuem para maior tendência de exposição ao risco de acidente de trânsito em motoristas idosos ${ }^{(7)}$. Alguns medicamentos usados por eles, como analgésicos, ansiolíticos ou antidepressivos, apresentam efeitos adversos sobre a direção veicular, pois agem direta ou indiretamente sobre o sistema nervoso central, afetando a função psicomotora e/ou equilíbrio ${ }^{(8)}$. A fragilidade física se traduz por alterações relacionadas à senescência como as neuromusculares, desregulação do sistema neuroendócrino e disfunção do sistema imunológico. Do mesmo modo, associa-se a um ciclo fisiológico de exacerbação do declínio de múltiplos sistemas, que resulta em diminuição da força muscular e tolerância ao esforço, déficit de energia e sarcopenia, levando o idoso a um estado de vulnerabilidade ${ }^{(9-10)}$.

O conteúdo do presente artigo é fruto da primeira etapa de um projeto de pesquisa maior intitulado "Fragilidade em idosos e a habilitação para direção veicular", que mostra resultados significativos para a sequência das investigações, sobretudo em razão do déficit encontrado na literatura vigente sobre a temática. O estudo é inédito na área gerontológica e desperta um novo contexto para o cuidado de enfermagem gerontológica.

Diante do exposto, o estudo objetivou rastrear as características dos idosos que realizam o exame de aptidão física e mental para a Carteira Nacional de Habilitação (CNH).

\section{METODOLOGIA}


Trata-se de estudo do tipo quantitativo descritivo e retrospectivo realizado com dados secundários obtidos dos registros do formulário padrão do órgão responsável pela habilitação para dirigir veículos automotores. O estudo foi realizado em uma clínica credenciada para o exame de aptidão física e metal para direção veicular, na cidade de Curitiba-Paraná. As clínicas credenciadas no município de Curitiba perfazem 54 unidades (quantitativo do período de realização da pesquisa). A seleção da unidade ocorreu por sorteio aleatório simples, de acordo com a lista de clínicas fornecida pelo órgão que as credencia.

A distribuição dos idosos por clínicas segue a Resolução do Conselho Federal de Medicina (CFM) $n^{0}$ 1636/2002(11), a qual estabelece distribuição imparcial de todos os exames pela divisão equitativa obrigatória, aleatória e impessoal entre as entidades e médicos credenciados, sendo a distribuição dos exames realizada pelo Departamento de Trânsito e nunca por escolha do periciado, entre as clínicas credenciadas do município.

O estudo foi desenvolvido com dados registrados no formulário padrão sobre idosos de 60 anos ou mais que se submeteram ao exame de aptidão física e mental e/ou à avaliação psicológica para obtenção, renovação, adição ou mudança de categoria para conduzir veículos automotores. O período amostral foi de dezembro de 2012 a dezembro de 2014 e a amostra constituída por 1023 formulários. Estabeleceram-se os seguintes critérios de inclusão dos idosos no estudo: realizar o exame de aptidão física e mental na clínica; e apresentar idade igual ou superior a 60 anos completos até a data de realização do exame. Não houve critérios de exclusão.

A coleta de dados iniciou em outubro de 2014 e foi concluída em março de 2015, por meio de instrumento do tipo formulário, embasado nas variáveis contidas no Registro Nacional de Condutores Habilitados (RENACH), composto pelas seguintes variáveis de interesse: sexo, idade, ocupação, medicamentos, deficiência física, doenças (psiquiátricas, cardíacas, oftalmológicas, locomotoras, auditivas, de membros superiores, neurológica e do equilíbrio postural), dados antropométricos, dinamometria, drogas ilícitas, acidente de trânsito e aptidão para dirigir.

Os dados foram organizados no programa computacional Excel ${ }^{\circledR} 2007$ e analisados no software Statistical Package for the Social Sciences versão 21.0. Os dados foram tratados por meio de estatística descritiva, com distribuição de frequência absoluta e relativa, média e desvio padrão.

O projeto de pesquisa obteve parecer favorável com parecer $\mathrm{n}^{\circ}$ 833.460. O acesso à clínica credenciada, bem como ao Registro Nacional de Condutores Habilitados, foi realizado por meio de autorização e parceria firmada com as clínicas e órgão responsável pela habilitação para dirigir veículos automotores.

\section{RESULTADOS}

Na Tabela 1, visualiza-se a predominância de idosos do sexo masculino ( $n=713 ; 69,7 \%$ ), na faixa etária entre 60 e 64,9 anos $(n=418 ; 40,9 \%$ ). A idade média dos idosos foi de $67 \pm 6,1$ anos, sendo $67,4 \pm 6,3$ anos para o sexo masculino e $66 \pm 5,6$ anos para o sexo feminino. Quanto às variáveis clínicas, a maioria dos idosos relatou o uso de um ou mais medicamentos $(n=621 ; 60,7 \%)$, mas não declarou possuir problemas de saúde ( $n=861 ; 84,2 \%)$, igualmente, a maioria nega deficiência física ( $n=990 ; 96,8 \%)$, episódio de tontura $(n=1009 ; 98,6 \%)$ e acidente de trânsito $(n=878 ; 85,8 \%)$. De acordo com a categorização dos valores de IMC(12), os registros apontaram 419 idosos (41\%) com sobrepeso e 195 (19\%) obesidade.

Tabela 1 - Distribuição das características sociodemográficas e clínicas dos idosos submetidos ao exame de aptidão física e mental para a carteira nacional de habilitação. Curitiba, PR, Brasil, 2015 (continua)

\begin{tabular}{lll} 
CARACTERÍSTICAS & CLASSIFICAÇÃO & $\mathbf{n}(\mathbf{\%})$ \\
\hline Sexo & Feminino & $310(30,3)$ \\
\cline { 2 - 3 } & Masculino & $713(69,7)$ \\
\hline
\end{tabular}




\begin{tabular}{|c|c|c|}
\hline \multirow[t]{5}{*}{ Idade } & $60-64,9$ anos & $418(40,9)$ \\
\hline & $65-69,9$ anos & $329(32,2)$ \\
\hline & $70-74,9$ anos & $137(13,4)$ \\
\hline & $75-79,9$ anos & $91(8,9)$ \\
\hline & 80 anos ou mais & $48(4,7)$ \\
\hline \multirow[t]{4}{*}{ Profissão/ Setor } & Primáriot & $6(0,6)$ \\
\hline & Secundário \pm & $63(6,2)$ \\
\hline & Terciário§ & $624(60,9)$ \\
\hline & Aposentados & $330(32,3)$ \\
\hline \multirow[t]{2}{*}{ Uso de medicamentos } & Sim & $621(60,7)$ \\
\hline & Não & $402(39,3)$ \\
\hline \multirow[t]{2}{*}{ Tontura } & Sim & $14(1,4)$ \\
\hline & Não & $1009(98,6)$ \\
\hline \multirow[t]{2}{*}{ Doenças } & Sim & $162(15,8)$ \\
\hline & Não & $861(84,2)$ \\
\hline \multirow[t]{2}{*}{ Acidente de trânsito } & Sim & $145(14,2)$ \\
\hline & Não & $878(85,8)$ \\
\hline \multirow[t]{5}{*}{ Índice de Massa Corpórea (IMC) } & Baixo peso & $22(2,2)$ \\
\hline & Eutrófico & $248(24,2)$ \\
\hline & Sobrepeso & $419(41)$ \\
\hline & Obesidade & $195(19,1)$ \\
\hline & Não respondeu & $139(13,5)$ \\
\hline
\end{tabular}

Fonte: Os autores (2015)

†Setor primário = agronegócios e extrativismo mineral. \pm Setor secundário = indústria de transformação e construção civil. $\S$ Setor terciário = serviços em geral e comércio ${ }^{(13)}$.

Quanto à variável acuidade visual, $573(56 \%)$ idosos utilizavam tecnologias assistivas para correção visual. As demais variáveis do exame de aptidão física e mental (acuidade auditiva, ausculta cardíaca, avaliação de membros superiores e inferiores) não apresentaram alterações significativas. Observouse prevalência de idosos com dinamometria direita e esquerda equivalente a 30-40 Kgf ( $n=975 ; 95,3 \%$ e $\mathrm{n}=971 ; 94,9 \%$, respectivamente) (Tabela 2 ).

Tabela 2 - Distribuição dos resultados dos idosos submetidos ao exame de aptidão física e mental para a carteira nacional de habilitação. Curitiba, PR, Brasil, 2015 (continua)

\begin{tabular}{llc} 
CARACTERÍSTICAS & CLASSIFICAÇÃO & n (\%) \\
\hline Acuidade visual & Com correção & $573(56)$ \\
\cline { 2 - 3 } & Sem correção & $444(43,4)$ \\
\cline { 2 - 3 } & Não respondeu & $6(0,6)$ \\
\hline Acuidade auditiva & Alterada & $0(0)$ \\
\cline { 2 - 3 } & Normal & $1021(99,8)$ \\
\cline { 2 - 3 } & Não respondeu & $2(0,2)$ \\
\hline Ausculta cardiológica & Alterada & $48(4,7)$ \\
\cline { 2 - 3 } & Normal & $970(94,8)$ \\
\cline { 2 - 3 } & Não respondeu & $5(0,5 \%)$ \\
\hline Membros superiores & Alterada & $16(1,6)$ \\
\cline { 2 - 3 } & Normal & $1005(98,2)$ \\
\cline { 2 - 3 } & Não respondeu & $2(0,2 \%)$ \\
\hline
\end{tabular}




\begin{tabular}{llc}
\hline Dinamometria direita & \multicolumn{1}{l}{$10-20 \mathrm{kgf}$} & $42(4,1 \%)$ \\
\cline { 2 - 3 } & $30-40 \mathrm{kgf}$ & $975(95,3)$ \\
\cline { 2 - 3 } & Não respondeu & $6(0,6 \%)$ \\
\hline Dinamometria esquerda & $10-20 \mathrm{kgf}$ & $44(4,3 \%)$ \\
\cline { 2 - 3 } & $30-40 \mathrm{kgf}$ & $971(94,9)$ \\
\cline { 2 - 3 } & Não respondeu & $18(0,8 \%)$ \\
\hline Membros inferiores & Alterada & $1001(97,8)$ \\
\cline { 2 - 3 } & Normal & $4(0,4 \%)$ \\
\cline { 2 - 3 } & Não respondeu & 100
\end{tabular}

Fonte: Os autores (2015)

No resultado final do exame de aptidão física e mental dos idosos (Gráfico 1), 216 (21,1\%) foram considerados aptos, $803(78,5 \%)$ aptos com uma ou mais restrições para dirigir e $4(0,4 \%)$ inaptos temporários.

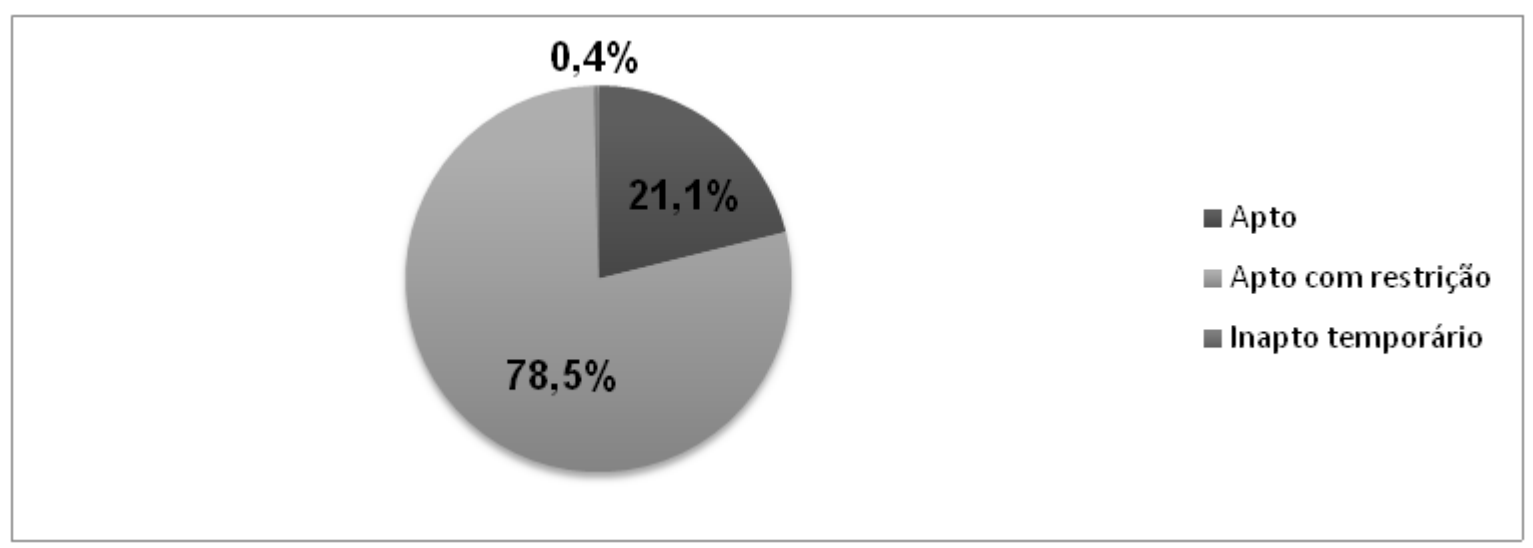

Gráfico 1 - Distribuição do resultado final dos idosos no exame de aptidão física e mental para a carteira nacional de habilitação. Curitiba, PR, Brasil, 2015

Dos 803 idosos aptos com restrição, a maior distribuição foi para o uso de lentes corretivas ( $\mathrm{n}=560$; $54,7 \%)$, seguida pela categoria não autorizada C ( $n=374 ; 36,6 \%$ ) (Tabela 3 ).

Tabela 3 - Distribuição das restrições dos idosos no exame de aptidão física e mental para a carteira nacional de habilitação. Curitiba, PR, Brasil, 2015

\begin{tabular}{llc} 
CARACTERÍ́STICASt & CLASSIFICAÇÃO & (\%) \\
\hline Uso de lentes corretivas & Sim & $560(54,7)$ \\
\hline Categoria não autorizada & Somente C & $374(36,6)$ \\
\cline { 2 - 3 } & A e C & $17(1,7)$ \\
\hline Uso de prótese auditiva & Sim & $18(1,8)$ \\
\hline Visão monocular & Sim & $10(1)$ \\
\hline Vedado dirigir em rodovias e vias de trânsito rápido & Sim & $6(0,6)$ \\
\hline Vedado dirigir após o pôr do sol & Sim & $2(0,2)$ \\
\hline Condutor surdo & Sim & $0(0)$
\end{tabular}

+Cada idoso pode apresentar uma ou mais restrições para dirigir.

Fonte: Os autores (2015) 


\section{- DISCUSSÃO}

Houve predominância de idosos do sexo masculino, na faixa etária de 60-64,9 anos, profissionais do setor terciário, seguido de aposentados e pensionistas. Apesar de estudos apontarem a feminização da velhice ${ }^{(14-15)}$, observa-se que o número de idosos do sexo masculino condutores de veículos é duas vezes maior do que o observado no sexo feminino. Tal resultado está de acordo com as estatísticas do Departamento de Trânsito do Paraná(16), que revelaram maior número de idosos do sexo masculino condutores de veículos em Curitiba ( $\mathrm{n}=108.519)$, quando comparado ao sexo feminino $(\mathrm{n}=49.037)$.

O número de homens habilitados para direção veicular é predominante em todas as faixas etárias ${ }^{(16)}$. Esse resultado está relacionado à maior inserção do homem no mercado de trabalho e às profissões predominantemente exercidas por eles, como, por exemplo, a de motorista de veículos. As mulheres idosas de hoje, com maior frequência, foram vítimas de discriminação no acesso à educação, salário, alimentação, trabalho significativo e poder político ${ }^{(17)}$. Essas desvantagens cumulativas também podem ter influenciado o menor acesso das idosas à carteira de motorista.

Os idosos jovens, no presente estudo, mostraram-se ativos, com capacidade funcional preservada e com menores índices de fragilidade física, o que justifica o maior número desses no setor de habilitação para direção veicular. Segundo estudo internacional ${ }^{(18)}$, os problemas de saúde são as razões mais comuns para o idoso parar de dirigir, seguidos por conselhos médicos, acidentes de trânsito, intervenções familiares ou influência de algumas variáveis, como a idade.

Houve predominância de idosos que referiram utilizar medicamentos e não possuir problemas de saúde. Sabe-se que o uso de medicamentos aumenta com a idade e este dado converge ao encontrado na literatura internacional. Nos EUA, idosos acima de 65 anos consomem mais de $30 \%$ das prescrições anuais de medicamentos ${ }^{(8)}$. O fato dos idosos conduzirem veículos automotores em idades cada vez mais avançadas, em comparação as gerações anteriores, suscita a necessidade de se desenvolver estudos mais aprofundados de como os medicamentos afetam a capacidade de condução no idoso ${ }^{(19)}$. Enquanto é conhecido que o álcool é um fator contribuinte para as colisões de veículos automotores, a contribuição dos medicamentos nos acidentes de trânsito ainda não é bem explicitada ${ }^{(19)}$.

O uso de medicamento contínuo para o tratamento de algumas doenças, e consequentemente controle dos sintomas, faz com que alguns idosos não percebam a presença da doença em seu cotidiano, o que pode justificar, parcialmente, o resultado encontrado.

O sintoma tontura foi relatado por uma minoria de idosos. Em Natal-RN foi realizado um estudo que avaliou a presença de tontura em 50 idosos, na faixa etária entre 60 a 88 anos, participantes de um grupo de convivência do Sistema Único de Saúde (SUS). Os resultados mostram que 17 (35,1\%) dos idosos apresentaram vertigem, 6 (13,5\%) vertigem associada a outro tipo de tontura, 12 (24,3\%) desequilíbrio, 4 (8,1\%) flutuação, 8 (16,2\%) pré-síncope e 1 (2,8\%) flutuação e pré-síncope ${ }^{(20)}$.

O baixo percentual de idosos com episódios de tontura e síncope é esperado, já que estes eram idosos que se submeteram ao exame de aptidão física e mental para a carteira nacional de habilitação veicular e a presença desses sintomas poderia inviabilizar a direção veicular. Segundo o Conselho Nacional de Trânsito ${ }^{(21)}$, órgão máximo normativo e consultivo do Sistema Nacional de Trânsito, quando o sintoma tontura é relatado pelo candidato à $\mathrm{CNH}$, o médico perito examinador de trânsito deverá solicitar exame otoneurológico para avaliação da condição de segurança para direção veicular - essa é razão suficiente para justificar a baixa prevalência na população analisada.

Entre os 1023 idosos avaliados, 573 (56,0\%) deles apresentaram necessidade de correção visual. O ato de dirigir exige do motorista uma boa acuidade visual, com a utilização ou não de lentes corretivas. Estudo de coorte realizado com 2520 idosos norte americanos, cujo objetivo foi associar os tipos de perda de função visual com as alterações na condução veicular, mostrou que a variável acuidade visual está associada significativamente às variáveis redução de quilômetros percorridos e dirigir em áreas desconhecidas ${ }^{(22)}$. A diminuição da função visual indica alterações na maneira que os idosos conduzem os veículos automotores e a avaliação da acuidade visual é de extrema importância nessa faixa etária.

Houve predominância de idosos $(n=1021 ; 99,8 \%)$ que apresentaram acuidade auditiva normal e este dado se contrapõe ao encontrado na literatura. $\mathrm{O}$ estudo ${ }^{(23)}$ realizado em uma Universidade Aberta à 
Terceira Idade na cidade de Salvador (BA) avaliou a audição de 40 idosos com idade entre 61 e 88 anos. Por meio do Inventário Auditivo para idosos (IAPI) e exame audiométrico, o estudo mostrou que 37,5\% dos idosos apresentavam audição normal e 62,5\% alguma perda auditiva, sendo 30\% perda auditiva assimétrica e 32,5\% simétrica. Sabe-se que a capacidade de audição é item indispensável para a boa condução veicular e, portanto, são necessárias medidas como exclusão do autorrelato e exames mais sensíveis.

A avaliação cardiorrespiratória realizada através da ausculta cardíaca apresentou alterações em 48 $(4,7 \%)$ idosos, entre elas arritmias e bulhas cardíacas hipofonéticas. Entretanto, sabe-se que o percentual de alterações cardíacas em idosos brasileiros é maior do que o resultado encontrado. Inquérito realizado entre idosos de 16 capitais brasileiras obteve que $12,6 \%$ possuíam doença isquêmica do coração associada à hipertensão arterial sistêmica e ao diabetes mellitus ${ }^{(24)}$.

O Consenso da Associação Brasileira de Medicina do Tráfego (ABRAMET) estabelece que o Infarto do Miocárdio (IM) leva a inaptidão temporária para a direção veicular por oito semanas, enquanto que a Angina Pectoris apenas quando tiver os sintomas controlados, podendo em ambas as patologias o prazo de renovação ser reduzido a critério médico. A Insuficiência Cardíaca (IC) torna o candidato inapto temporariamente até a estabilização do quadro ${ }^{(25)}$.

Quanto à força dos membros superiores e inferiores, a maioria dos idosos ( $\mathrm{n}=1005 ; 98,2 \%$ e $n=1001 ; 97,8 \%$, respectivamente) foi considerado dentro da normalidade. A força dos membros inferiores não é avaliada no exame de aptidão física e mental segundo padrões estabelecidos pela literatura vigente. Estudos nacionais ${ }^{(17,26)}$ e internacionais ${ }^{(27-28)}$ apontam que o aumento da idade implica em diminuição da velocidade da marcha. A diminuição da velocidade da marcha influencia na vitalidade dos idosos, pois sua execução, controle de movimento e apoio está relacionada ao funcionamento de múltiplos órgãos, como coração e pulmão, sistema circulatório, nervoso e músculo esquelético ${ }^{(27)}$.

É de extrema importância a aplicação de testes de desempenho simples, como a avaliação da velocidade da marcha em idosos que dirigem. Tais testes permitem a identificação do declínio funcional precoce e o rastreamento de idoso com perda de massa muscular e, consequentemente, da força dos membros inferiores para utilizar os pedais.

A avaliação da força de preensão manual mostrou valores entre $30 \mathrm{kgf}$ ( $\mathrm{n}=975 ; 95,3 \%$ ) e 40 kgf $(\mathrm{n}=971 ; 94,9 \%)$ para membro superior direito e esquerdo respectivamente. Os valores de FPM são compatíveis com os exigidos no exame de direção veicular (20 kgf em cada uma das mãos para candidatos à direção de veículos das categorias moto e carro e de $30 \mathrm{kgf}$ candidatos à direção de veículos das categorias caminhões de transporte de carga, de passageiro e com reboque) com médias relativamente altas ao comparadas aos demais idosos da comunidade.

Os resultados de FPM são indicativos de que esses idosos se mostram ativos e robustos, com força muscular compatível com a renovação da $\mathrm{CNH}$. Deve-se levar em conta o tipo de dinamômetro utilizado na mensuração, considerado menos preciso quando comparado ao utilizado em estudos nacionais e internacionais.

No presente estudo, observou-se predominância de idosos com sobrepeso. Tal dado é preocupante, visto que o aumento excessivo do peso é fator de risco para várias doenças, principalmente as relacionadas ao coração. De acordo com estudos ${ }^{(29-30)}$, a obesidade aumenta o risco de lesão grave em acidentes de carro. $\mathrm{O}$ aumento do IMC causa alterações anatômicas e fisiológicas que modificam a resposta normal dos ocupantes em relação ao cinto de segurança durante um acidente ${ }^{(29)}$. Dessa maneira, o aumento da transferência de energia e impulso pode substituir o efeito de proteção da camada adiposa e causar ferimentos mais graves ${ }^{(30)}$.

A partir do resultado obtido, sugere-se que outros estudos sejam realizados com enfoque no IMC dos ocupantes de veículos. Tais investigações podem contribuir para a concepção de novos equipamentos de segurança, que se adaptem aos diferentes níveis de IMC e resultem na diminuição da gravidade das lesões sofridas por motoristas e passageiros.

Quanto aos resultados finais do exame para direção veicular, observa-se que houve predomínio de idosos classificados como aptos com uma ou mais restrições para dirigir. Os idosos que se submetem ao exame de aptidão para direção veicular apresentam baixo nível de reprovação. Salienta-se que as 
questões de autorrelato dirigidas aos candidatos, com itens relacionados aos fatores que interferem na direção veicular, são passíveis de omissão pelos candidatos.

Como limitação do estudo, ressalta-se que algumas variáveis de interesse que sustentariam outras discussões não foram tratadas, pois não constavam nos registros do formulário analisado. Sugere-se a realização de outras pesquisas do tipo transversal e longitudinal, com o intuito de explorar ainda mais as características e relações entre idoso e direção veicular.

\section{- CONCLUSÃO}

Os idosos motoristas são homens na faixa etária idoso - jovem, que utilizam medicamentos, lentes corretivas, não possuem deficiência física e não relatam tontura, desmaio, convulsão, vertigem e outras doenças. No exame físico, apresentaram acuidade auditiva preservada, ausculta cardiológica normal, membros superiores e inferiores sem alterações e dinamometria em ambos os membros superiores compatíveis com valores normais. Quanto à direção veicular, houve predomínio de idosos considerados aptos com algum tipo de restrição. $O$ uso de lentes corretivas e a categoria não autorizada $C$ foram as restrições predominantes.

O estudo é inédito na área de enfermagem gerontológica e com ele espera-se despertar o interesse dos profissionais de enfermagem para o contexto da direção veicular. Os resultados fornecem subsídios para o avanço da temática em estudos que têm por objetivo aprimorar as avaliações dos idosos e, consequentemente, contribuir para um trânsito mais seguro.

\section{APOIO FINANCEIRO}

Pesquisa financiada pela Fundação Araucária de Apoio ao Desenvolvimento Científico e Tecnológico do Paraná (CP 09/15: protocolo n²5784).

\section{REFERÊNCIAS}

1. Centro Internacional de Longevidade Brasil (ILC-Brasil). Envelhecimento ativo: um marco político em resposta à evolução da longevidade. [Internet] 2015 [acesso em 23 ago 2016]. Disponível: http://ilcbrazil.org/portugues/ wp-content/uploads/sites/4/2016/02/Envelhecimento-Ativo.pdf

2. Great Britain - Department For Transport (DFT). Statistical Release - National Travel Survey 2012. [Internet] 2013 [acesso em 20 fev 2016]. Disponível: https://www.gov.uk/government/uploads/system/uploads/attachment_ data/file/243957/nts2012-01.pdf

3. Governo do Estado do Paraná (PR). Anuário estatístico: 2013. Curitiba: Governo do Estado do Paraná; Departamento de Trânsito do Paraná; 2014. 134p. Relatório Final.

4. Marottoli RA, de Leon CFM, Glass TA, Williams CS, Cooney Jr LM, Berkman LF. Consequences of driving cessation: Decreased out of home activity levels. J Gerontol B Psychol Sci Soc Sci. [Internet] 2000;55(6) [acesso em 22 fev 2016]. Disponível: https://www.ncbi.nlm.nih.gov/pubmed/11078110

5. Cunha UGV, Thomaz DP. Risk of dementia misdiagnosis among car drivers. Rev Med Minas Gerais. [Internet] 2011;21(2) [acesso em 22 fev 2016]. Disponível: http://pesquisa.bvsalud.org/ses/resource/pt/lil-598711

6. Lacerda LP, Carlos CMG. O idoso no trânsito. UNAR. [Internet] 2012;6(1) [acesso em 14 fev 2016]. Disponível: http://revistaunar.com.br/cientifica/documentos/vol6_n1_2012/4_o_idoso_no_transito.pdf

7. Mcgwin Jr G, Sims RV, Pulley L, Roseman JM. Relations among chronic medical conditions, medications, and automobile crashes in the Elderly: a population-based case-control study. Am J Epidemiol. [Internet] 2000;152(5) [acesso em 12 mar 2016]. Disponível: https://www.ncbi.nlm.nih.gov/pubmed/10981455

8. Rudisill TM, Zhu M, Davidov D, Leann Long D, Sambamoorthi U, Abate M, et al. Medication use and the risk of motor vehicle collision in West Virginia drivers 65 years of age and older: a case-crossover study. BMC Res Notes. 
9. Fried LP, Tangen CM, Walston J, Newman AB, Hirsch C, Gottdiener J, et al. Frailty in older adults: Evidence for a phenotype. J. Gerontol A Biol Sci Med Sci. [Internet] 2001;56(3) [acesso em 13 abr 2013]. Disponível: https://www. ncbi.nlm.nih.gov/pubmed/11253156

10. Zaslavsky O, Cochrane BB, Thompson HJ, Woods NF, Herting JR, LaCroix A. Frailty: a review of the first decade of research. Biol Res Nurs. [Internet] 2013;15(4) [acesso em 13 abr 2014]. Disponível: http://www.ncbi.nlm. nih.gov/pubmed/23086382

11. Conselho Federal de Medicina. Resolução $\mathrm{n}^{\circ} 1636$ de 10 de maio de 2002. Dispõe sobre o teste exame de aptidão física e mental para condutores de veículos automotores. Brasília: CFM; 2002.

12. Organización Panamericana de la Salud (OPAS). Encuesta Multicêntrica: Salud, bienestar y envejecimiento (SABE) en América Latina y el Caribe. XXXVI Reunión del Comité Asesor de Investigaciones en Salud. 2001 [acesso em 23 out 2016]. Disponível: http://envejecimiento.csic.es/documentos/documentos/paho-salud-01.pdf

13. Pochmannm M. Economia solidária no Brasil: possibilidades e limites. IPPEA [Internet] 2004;24(n esp) [acesso em 23 out 2016]. Disponível: http://repositorio.ipea.gov.br/handle/11058/5249?locale=pt_BR

14. Salgado CDS. Mulher idosa: a feminização da velhice. Estud. interdiscip. Envelhec. [Internet] 2002;4(n esp) [acesso em 16 de jul 2016]. Disponível: http://seer.ufrgs.br/index.php/RevEnvelhecer/article/view/4716/2642

15. de Lima LCV, Bueno CMLB. Envelhecimento e gênero: A vulnerabilidade de idosas no Brasil. Revista Saúde e Pesquisa. [Internet] 2009;2(2) [acesso em 04 dez 2015]. Disponível: http://periodicos.unicesumar.edu.br/index. php/saudpesq/article/view/1173

16. Governo do Estado do Paraná (BR). Anuário estatístico: 2012. Curitiba: Governo do Estado do Paraná; Departamento de Trânsito do Paraná; 2012. 134p. Relatório Final.

17. Lenardt MH, Carneiro NHK, Betiolli SE, Ribeiro DKMN, Wachholz PA. Prevalence of pre-frailty for the component of gait speed in older adults. Rev. Latino-Am. Enfermagem. [Internet] 2013;21(3) [acesso em 10 Abr 2016]. Disponível: http://dx.doi.org/10.1590/S0104-11692013000300012

18. Chihuri S, Mielenz TJ, DiMaggio CJ, Betz ME, DiGuiseppi C, Jones VC, et al. Driving Cessation and Health Outcomes in Older Adults. J Am Geriatr Soc. [Internet] 2016;64(2) [acesso em 13 abr 2016]. Disponível: https:// www.ncbi.nlm.nih.gov/pubmed/26780879

19. Kelly E, Darke S, Ross J. A review of drug use and driving: epidemiology, impairment, risk factors and risk perceptions. Drug Alcohol Rev. [Internet] 2004;23(3) [acesso em 24 abr 2016]. Disponível: http://dx.doi.org/10.10 80/09595230412331289482

20. Ferreira LMBM, Ribeiro KMOBF, Pestana ALS, de Lima KC. Prevalence of dizziness in older people. Rev. CEFAC. [Internet] 2014;16(3) [acesso 17 jul 2015]. Disponível: http://dx.doi.org/10.1590/1982-021620142913

21. Conselho Nacional de Trânsito. Resolução n 425 de 27 de novembro de 2012. Dispõe sobre o exame de aptidão física e mental, a avaliação psicológica e o credenciamento das entidades públicas e privadas de que tratam o art. 147, I e $\S \S 1^{\circ}$ a $4^{\circ}$ e o art. 148 do Código de Trânsito Brasileiro. Brasília: Contran; 2012.

22. Freeman EE, Munõz B, Turano KA, West SK. Measures of Visual Function and Their Association with Driving Modification in Older Adults. IOVS. [Internet] 2006;47(2) [acesso em 26 abr 2016]. Disponível: http://dx.doi. org/10.1167/iovs.05-0934

23. de Sousa MGC, Russo ICP. Hearing and perception of hearing loss in elderly people. Rev. soc. bras. fonoaudiol. [Internet] 2009;14(2) [acesso em 15 jul 2015]. Disponível: http://dx.doi.org/10.1590/S1516-80342009000200016

24. Pereira JC, Barreto SM, Passos VMA. The profile of cardiovascular health of elderly Brazilian people needs to improve: a population-based study. Arq. Bras. Cardiol. [Internet] 2008;91(1) [acesso em 10 mai 2015]. Disponível: http://dx.doi.org/10.1590/S0066-782X2008001300001

25. Conselho Nacional de Trânsito. Resolução $n^{\circ} 267$ de 15 de fevereiro de 2008. Dispõe sobre o exame de aptidão física e mental, a avaliação psicológica e o credenciamento das entidades públicas e privadas de que tratam o art. 147, I e $\S \S 1^{\circ}$ a $4^{\circ}$ e o art. 148 do Código de Trânsito Brasileiro. Brasília: Contran; 2008. 
26. Busch TA, Duarte YA, Nunes DP, Lebrão ML, Naslavsky MS, Rodrigues AS, et al. Factors associated with lower gait speed among the elderly living in a developing country: a cross-sectional population-based study. BMC Geriatrics. [Internet] 2015;15 [acesso em 23 de out 2016] Disponível: http://dx.doi.org/10.1186/s12877-015-0031-2

27. Studenski S, Perera S, Patel K, Rosano C, Faulkner K, Inzitari M, et al. Gait speed and survival in older adults. JAMA. [Internet] 2011;305(1) [acesso em 23 mai 2014]. Disponível: http://www.ncbi.nlm.nih.gov/pmc/articles/ PMC3080184/

28. Jankovik J. Gait disorders. Neurol Clin. [Internet] 2015;33(1) [acesso em 14 abr 2016]. Disponível: http://dx.doi. org/10.1016/j.ncl.2014.09.007

29. Zhu S, Kim JE, Ma X, Shih A, Laud PW, Pintar F, et al. BMI and Risk of Serious Upper Body Injury Following Motor Vehicle Crashes: Concordance of Real-World and Computer-Simulated Observations. PLoS Med. [Internet] 2010;7(3) [acesso em 13 abr 2016]. Disponível: http://dx.doi.org/10.1371/journal.pmed.1000250

30. Carter PM, Flannagan CA, Reed MP, Cunningham RM, Rupp JD. Comparing the Effects of Age, BMI and Gender on Severe Injury (AIS 3+) in Motor-Vehicle Crashes. Accid Anal Prev. [Internet] 2014;72(n esp) [acesso em 14 abr 2016]. Disponível: http://www.ncbi.nlm.nih.gov/pubmed/25061920 